\title{
LARGE EDDY SIMULATION OF TURBULENT INCOMPRESSIBLE FLUID FLOWS BY A NINE-NODES CONTROL VOLUME-FINITE ELEMENT METHOD
}

\author{
J. B. C. Silva ${ }^{a}$, \\ S. S. Mansurb, \\ and R. C. Lima \\ Universidade Estadual Paulista \\ Departamento de Engenharia Mecânica \\ Avenida Brasil, 56, CEP 15385-000 \\ C.P. 31, Ilha Solteira, SP, Brazil \\ ajbcampos@feis.unesp.br \\ bmansur@feis.unesp.br \\ crosiane@ita.br

\begin{abstract}
The main purpose of this work is the numerical computation of turbulent incompressible fluid flows by a nine-node control volume finite element method (CVFEM) using the methodology of large-eddy simulation.. The domain is discretized using nine nodes finite elements and the equations are integrated into control volumes around the nodes of the finite elements. The Navier Stokes equations are filtered for simulation of the large scales variables and the sub-grid scales stress appearing due to the filtering process are modeled through the eddy viscosity model of Smagorinsky. The two-dimensional benchmark problem of the lid-driven cavity flow is solved to validate the numerical code and preliminary results for the horizontal and vertical velocity profiles at the centerlines of the cavity and the stream functions are presented and compared with available results from the literature.

Keywords: Finite element, control volume, incompressible flow, large eddy simulation
\end{abstract}

\section{NOMENCLATURE}

Cs constant of Smagorisky

$C_{\alpha \beta} \quad$ coefficients of the convection matrix

$D \quad$ domain

$D_{i \alpha \beta} \quad$ coefficients of the continuity matrix

$F_{i \alpha}$

$f$

G

$H_{i \alpha \beta}$

K

$L$

$M_{\alpha \beta}$

$n_{j}$

nnel

nnep

$N_{\alpha}$

$N_{\beta}$

$p$

$P_{\alpha}$

$P_{\beta}$

Re

$S$

$S_{i}$

$\bar{S}_{i j}$

$S_{i \alpha \beta}$ coefficients of the source term vector function

filter function

coefficients of the pressure term matrix turbulent kinetic energy

characteristics length

coefficients of the mass matrix component of the unit outward normal vector

number of nodes of the linear elements number of nodes of the quadratic elements

interpolation function

dimensional pressure

nodal pressure

nodal pressure

Reynolds number

surface area

source term (vector)

rate of deformation

coefficients of the diffusive matrix interpolation function $t \quad$ time

$u_{i}, u_{j} \quad$ velocity component

$U_{i \alpha} \quad$ nodal velocity component

$U_{i \beta} \quad$ nodal velocity component

$V \quad$ volume

$x_{i}, X_{i \alpha} \quad$ coordinate components

$y_{i}, y \quad$ coordinate components

\section{Greek symbols}

$\Delta \quad$ Filter width

$\lambda$ time interpolation coefficient

$\mu \quad$ dynamical viscosity

$\mu_{t} \quad$ eddy viscosity

$\rho$ density

$\tau_{i j} \quad$ sub-grid scale stress tensor

$\Psi \quad$ stream function

\section{Subscripts}

in unit cell inlet

$w \quad$ tube surface

$\infty \quad$ free stream

\section{Superscripts}

$\begin{array}{ll}m & \text { maximum } \\ \text { opt } & \text { optimal }\end{array}$




\section{INTRODUCTION}

Fluid flows studies are of interest in many engineering applications and in the nature. Fluid flows are generally turbulent, occurring in complex domains and governed by a set of non-linear partial differential equations of convection-diffusive type. So, practical solutions must be obtained through numerical techniques such as: The Finite Difference Method (FDM), the Finite-Volume Method (FVM) and/or the Finite Element Method (FEM). The finite volume method is the most popular method to calculate fluid flows and one of its characteristics is that the terms in the equations have physical interpretation as fluxes, sources and so on, due to the conservative form of the equations.

In the last decades, the finite element method, originally, developed for structural problems, has been improved for using in Computational Fluid Dynamics (CFD) constituting a powerful tool to simulate actual situations of fluid flows by its versatility to discretize complex geometries. Baliga and Patankar (1980) introduced a hybrid numerical method named control volumefinite element method. The control volume-finite element method (CVFEM) combines attractive characteristics from both the finite element and the finite volume methods. In previous works, Silva and Moura (1997; 2001), Silva et al. (1998; 1999) and Silva (1998) developed a control volume finite element method using a quadratic, quadrilateral nine-noded element to simulate unsteady, incompressible and viscous fluid flows. In those works, no turbulence model was considered, so the results were obtained for relatively low Reynolds numbers.

Turbulent flows can be simulated through direct numerical simulation (DNS) of the NavierStokes equations, by using turbulence models (algebraic, two-equation models, second order models) with the Reynolds Average Navier Stokes equations (RANS), or by simulating the large scales variables after filtering the Navier-Stokes equations and modeling the sub-grid-scales stresses in the called large-eddy simulation (LES) methodology. DNS is very cost effective due to the grid and time refinements required to capture all length and time scales of turbulence. According to Wilcox (1993), the number of grid points and time-steps for a DNS of a flow in a channel to reach a steady state is of the order of $6.7 \times 10^{6}$ points and 32,000 time-steps, for a Reynolds number of 12,300. Two-equation models are of more amenable costs and large eddy simulation occupies an intermediate position between DNS and two-equation models. In LES, for the same Reynolds of 12,300, about $6.1 \times 10^{5}$ grid points in a channel would be required. With the increasing of the computational power, the use of LES to simulate fluid flows has gained many adepts.

In this work a large eddy simulation by using the CVFEM developed by Silva (1998) is presented. The benchmark problem of the liddriven cavity flow is solved and the results are compared with results obtained by other numerical techniques.

\section{Mathematical Expressions}

The unsteady, incompressible, viscous Newtonian flows are governed by the momentum and the continuity equations (Navier-Stokes equations). In LES, the equations are filtered for computation of the large-scale variables and the small scales computed are of the size of the grid. There is a vast literature about turbulence and LES has been increasingly used to simulate turbulent flows, mainly, due to the higher power and speed of computers available nowadays to many researchers. It is not the scope of this work to do a literature review on large eddy simulation but to implement a LES with a CVFEM of Silva (1998). Some excellent texts on LES were edited by Galperin and Orszag (1993). In Brazil, some groups at universities and research centers have published many works on this subject. A few works the authors of this article have consulted were Matos et al. (1999), Padilla and Silveira_Neto (2001), Almeida (2001) and Campregher (2002).

The filtered Navier-Stokes equations can be cast in the general form for components of velocity $u_{i}$ and pressure $p$ :

$$
\begin{aligned}
& \frac{\partial\left(\rho \bar{u}_{i}\right)}{\partial t^{*}}+\frac{\partial\left(\rho \bar{u}_{j} \bar{u}_{i}\right)}{\partial x_{j}}= \\
& -\frac{\partial \bar{p}}{\partial x_{i}}+\frac{\partial}{\partial x_{j}}\left(\mu \frac{\partial \bar{u}_{i}}{\partial x_{j}}\right)-\frac{\partial \tau_{i j}}{\partial x_{j}}+\bar{S}_{i}
\end{aligned}
$$




$$
\frac{\partial \bar{u}_{i}}{\partial x_{i}}=0
$$

where $\rho$ and $\mu$ are the density and the dynamic viscosity respectively, $S_{i}$ is a source term accounting for the other terms not explicitly appearing in Eq. (1). Besides,

$$
\bar{f}(\vec{x})=\int_{D} f\left(\vec{x}^{\prime}\right) G\left(\vec{x}, \vec{x}^{\prime}, \Delta\right) d \vec{x}^{\prime}
$$

where an overbar denotes a filtered variable or large-scale variable on a domain $D ; G$ is a filter function, the most common are Gaussian, Top-hat or Sharp Fourier cut-off filters (Chidambaram, 1998); and $\Delta$ is the filter width, generally, $\Delta=\left(\Delta_{x} \Delta_{y} \Delta_{z}\right)^{1 / 3}$ in $3 \mathrm{D}$ and $\Delta=\left(\Delta_{x} \Delta_{y}\right)^{1 / 2}$ in 2D, with $\Delta_{x_{i}}$ being the grid size in the $x_{i}$-axis.

The sub-grid-scales (sgs) stress term $\tau_{i j}$ appearing in Eq. (1) results from the filter process. This term must be modeled, and is defined as:

$$
\tau_{i j}=\rho\left(\overline{u_{i} u_{j}}-\bar{u}_{i} \bar{u}_{j}\right)
$$

In this work the Smagorinsky model has been used and the sgs stress term is modeled in the form

$$
\tau_{i j}=\frac{2}{3} \rho k \delta_{i j}-2 \mu_{t}^{*} \bar{s}_{i j}
$$

where the eddy viscosity, $\mu_{t}$, the turbulent kinetic energy, $k$, and the deformation rate are respectively defined by the following expressions:

$$
\begin{aligned}
& \mu_{t}^{*}=\rho\left(C_{s} \Delta\right)^{2}\left(2 \bar{s}_{k l} \bar{s}_{k l}\right)^{1 / 2} ; k=\frac{\tau_{i i}}{2} ; \\
& \bar{s}_{i j}=\frac{1}{2}\left(\frac{\partial \bar{u}_{i}}{\partial x_{j}}+\frac{\partial \bar{u}_{j}}{\partial x_{i}}\right)
\end{aligned}
$$

In the simulations of the present work the constant of Smagorinsky was set to $C_{s}^{2}=0.026$ and the filter width was chosen to be equal to the length of control volume face where the convective and diffusive fluxes are considered:

$$
\left(\Delta=\int_{\xi} \sqrt{(\partial x / \partial \xi)^{2}+(\partial y / \partial \xi)^{2}} d \xi \quad\right. \text { or }
$$

$\left.\Delta=\int_{\eta} \sqrt{(\partial x / \partial \eta)^{2}+(\partial y / \partial \eta)^{2}} d \eta\right)$. See Fig. 2 for reference.

After the substitution of Eqs. (5) and (6) into Eq. (1), one obtains the following equations, now, in dimensionless form:

$$
\begin{aligned}
& \frac{\partial U_{i}}{\partial t}+\frac{\partial\left(U_{j} U_{i}\right)}{\partial X_{j}}=-\frac{\partial P_{t}}{\partial X_{i}}+ \\
& +\frac{\partial}{\partial X_{j}}\left(\left(\frac{1}{\operatorname{Re}}+v_{t}\right) \frac{\partial U_{i}}{\partial X_{j}}\right)+\frac{\partial}{\partial X_{j}}\left(v_{t} \frac{\partial U_{j}}{\partial X_{i}}\right)+F_{i}
\end{aligned}
$$

$$
\frac{\partial U_{i}}{\partial X_{i}}=0
$$

where the kinetic energy term was included in the pressure term, resulting the turbulent pressure, $P_{t}=\bar{P}+\frac{2}{3} \frac{k}{u_{0}^{2}}$. The dimensionless variables are defined as follows:

$$
\begin{aligned}
& X_{i}=\frac{x_{i}}{L} ; \quad U_{i}=\frac{\bar{u}_{i}}{u_{0}} ; \quad \bar{P}=\frac{\bar{p}-p_{0}}{\rho u_{0}^{2}} ; t=\frac{t^{*}}{L / u_{0}} ; \\
& v_{t}=\frac{v_{t}^{*}}{u_{0} L}=\left(C_{s} \frac{\Delta}{L}\right)^{2}\left(2 \bar{S}_{k l} \bar{S}_{k l}\right)^{1 / 2} ; \quad \operatorname{Re}=\frac{\rho u_{0} L}{\mu}
\end{aligned}
$$

\section{NUMERICAL METHOD}

The CVFEM was firstly presented by Baliga and Patankar $(1980 ; 1983)$ for triangular elements and later by Raw and Schneider (1986) for quadrilateral elements. Several authors have enhanced the CVFEM since then. Raw et al. (1985) applied the nine-noded element to solve heat conduction problems. Banaszek (1989) did a comparison of the Galerkin and CVFEM methods in diffusion problems using six-noded and ninenoded elements. Silva (1998) developed a solver 


\section{CIÊNCIA/SCIENCE}

based on the nine-noded finite element and a control volume formulation to simulate 2D transient, incompressible, viscous fluid flows. During and after the development of the work of Silva (1998), some results were presented without considering the turbulence effect. Now, we have done simulations considering the model of Smagorinsky for the eddy viscosity. Silva (1998) and Silva and Moura (2001) presented the procedure of application of the CVFEM.

The formulation of CVFEMs involves five basic steps (Saabas and Baliga, 1994a; 1994b): (1) discretization of the domain of interest into elements; (2) further discretization of the domain into control volumes that surround the nodes in the finite element mesh, as shown in Fig. 1;

(3) definition of element-based interpolation functions for variables and physical properties of the fluid; (4) derivation of algebraic equations by using the sub-domain weighted residual method; and (5) assembling of the element equations forming the global matrix and choice of a procedure to solve the system of algebraic equations. Each node in the finite element mesh is inside a control volume. An element and its respectively control volumes are shown in Fig. 2 , together with the faces with convective and diffusive fluxes. These faces are identified as integration points.

Integration of Eqs. (7) and (8) inside each of the nine control volumes in a finite element results in:

$$
\begin{gathered}
\int_{V} \frac{\partial U_{i}}{\partial t} d V+ \\
\oint_{S}\left(U_{j} U_{i}-\left(\frac{1}{\operatorname{Re}}+v_{t}\right) \frac{\partial U_{i}}{\partial X_{j}}\right) n_{j} d S+\int_{V} \frac{\partial P_{t}}{\partial X_{i}} d V= \\
\oint_{S} v_{t} \frac{\partial U_{j}}{\partial X_{i}} n_{j} d S+\int_{V} F_{i} d V \\
\int_{V} \frac{\partial U_{i}}{\partial X_{i}} d V=0
\end{gathered}
$$

with $S$ and $V$ denoting the surface area and the volume of a control volume around a node in the element, $n_{j}$ is the outward normal vector to the area of a control volume where there are convective and diffusive fluxes.

In order to transform the integrals of Eqs. (10) and (11) into algebraic equations, we have interpolated the variables and coordinates in the finite element sense, so we have defined:

$$
\begin{aligned}
& U_{i}=\sum_{\alpha=1}^{\text {nnep }} N_{\alpha} U_{i \alpha} \\
& P=\sum_{\alpha=1}^{\text {nnel }} N_{\alpha} P_{\alpha} \\
& X_{i}=\sum_{\alpha=1}^{\text {nnep }} N_{\alpha} X_{i \alpha}
\end{aligned}
$$

where $N_{\alpha}$ are interpolating functions in reference element in local coordinates (Dhatt and Touzot, 1984); $U_{i \alpha}$ are the velocity components; $P_{\alpha}$ is the pressure and $X_{i \alpha}$ are components of the coordinate system at nodes a of an element; nnep and nnel are the number of nodes of quadratic (parabolic) and linear elements respectively. In order to satisfy the Ladyzhenskaya-Babuska-Brezzi (LBB) condition, the velocity field is interpolated by quadratic functions (nine-node elements) and the pressure is interpolated by linear functions (four-node elements) in a so called mixed formulation. Substitution of Eqs. (12)-(14) into Eqs. (10) and (11) results:

$$
\begin{gathered}
M_{\alpha \beta} \dot{U}_{i \beta}+C_{\alpha \beta}\left(U_{i \beta}\right) U_{i \beta}-S_{i \alpha \beta} U_{i \beta}+ \\
+H_{i \alpha \beta} P_{\beta}=F_{i \alpha} \\
D_{i \alpha \beta} U_{i \beta}=0
\end{gathered}
$$

where $M_{\alpha \beta}, C_{\alpha \beta}, S_{i \alpha \beta}, H_{i \alpha \beta}, D_{i \alpha \beta}$ and $F_{i \beta}$ are coefficients of the mass matrix, the convection matrix, the diffusive matrix, the pressure term matrix, the continuity matrix and the source term vector, respectively, for an element. 


\section{CIÊNCIA/SCIENCE}

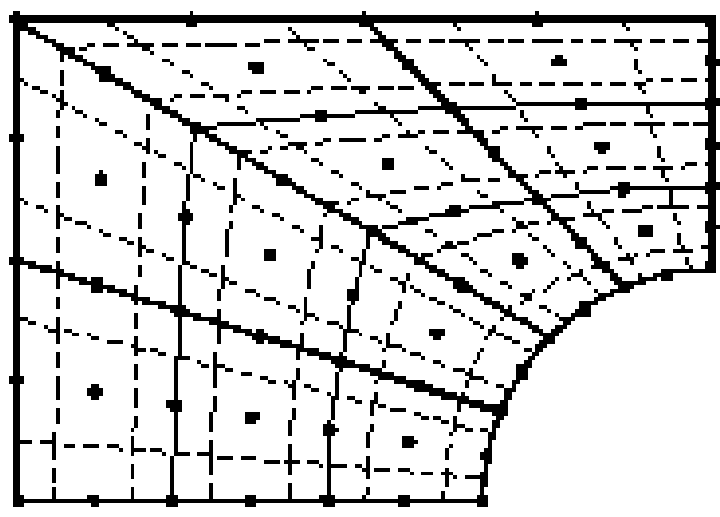

Figure 1. Meshes of finite elements and control volumes

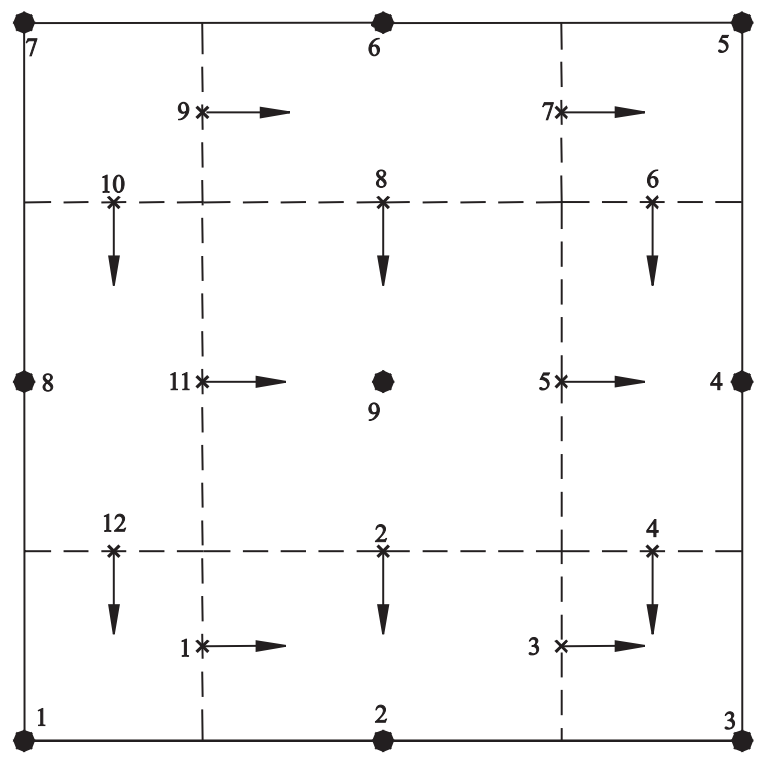

Figure 2. Element divided in control volumes and integration points

After the time discretization of Eqs. (15) and (16), one obtains the algebraic equations:

$$
\begin{gathered}
\frac{M_{\alpha \beta}}{\Delta t} U_{i \beta}^{n+1}+\lambda\left(C_{\alpha \beta}^{n+1}-S_{i \alpha \beta}\right) U_{i \beta}^{n+1}+ \\
+\lambda H_{i \alpha \beta} P_{\beta}^{n+1}=F_{i \alpha}^{n} \\
D_{i \alpha \beta} U_{i \beta}^{n+1}=0
\end{gathered}
$$

where

$$
\begin{aligned}
& F_{i \alpha}^{n}=\frac{M_{\alpha \beta}}{\Delta t} U_{i \beta}^{n}-(1-\lambda)\left(C_{\alpha \beta}^{n}-S_{i \alpha \beta}\right) U_{i \beta}^{n} \\
& -(1-\lambda) H_{i \alpha \beta} P_{\beta}^{n}
\end{aligned}
$$

and $0 \leq \lambda \leq 1$. If an implicit scheme is employed, $\lambda=1$, and in this case there is no need of aninitial condition for the pressure field.

The coefficient matrices are computed element by element in local coordinates shown in Fig. 3(a) and a global system of equations is assembled like in the classical finite element method.

(a)

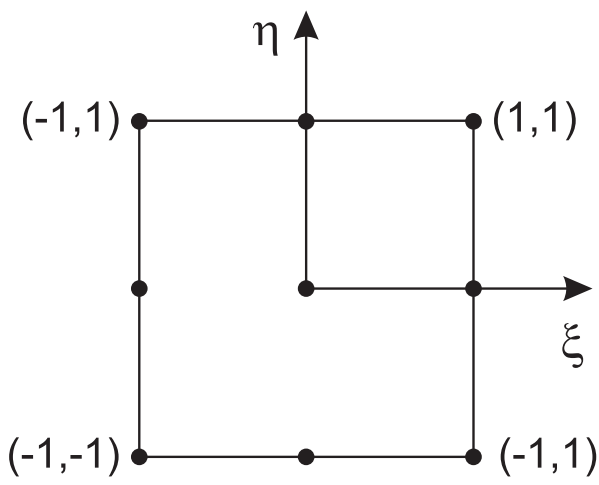

(b)

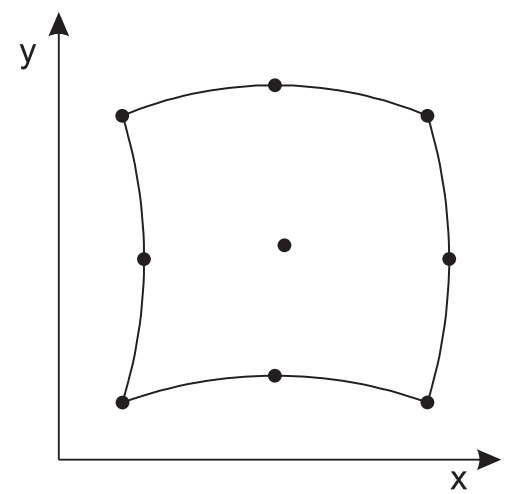

Figure 3. (a) Element in local coordinates $\xi-\eta$;

(b) Element in global coordinates $\mathrm{x}-\mathrm{y}$

The global system of equations was solved by the frontal method (Taylor and Hughes, 1981). In fact, the global system is never totally assembled in the computer memory, which is a characteristic of frontal method. So, personal computers may be used to solve the problems of interest. After the assembly, the global system of algebraic equations would have the form:

$$
K(U) U=F
$$

in which it can be seen that the global matrix $K$ depends on the global vector of unknowns $U$ due to the non-linearity of inertia terms. The linearization adopted has been that of successive substitution until the convergence is attained. Another way of linearization could be the Newton's method that converges fast but has a smaller radius 


\section{CIÊNCIA/SCIENCE}

of convergence than the successive approximation (Jiang, 1998).

\section{RESULTS AND DISCUSSION}

The simulations have been carried out for the classical benchmark problem of the lid-driven cavity flow. The geometry for this flow is very simple, however, this flow presents some interesting aspects like re-circulation zones that constitute a good challenge for simulation by numerical methods.

\section{LID-DRIVEN CAVITY FLOW}

The flow in a square cavity induced by the upper plate motion is considered as the benchmark problem. The velocities are set to zero at all walls, except the velocity along the $\mathrm{x}$-axis for the upper plate. The pressure was set to zero at the mid bottom point. Figures 4 and 5 show the results for $U$ velocity at $\mathrm{X}=0.5$ and $\mathrm{V}$ velocity at $\mathrm{Y}=0.5$ for Reynolds numbers of 100, 400, 1,000 and 10,000 of the present work compared with the results from Ghia et al. (1982), when the permanent regime is established. As can be seen in Figs. 4 and 5 the agreement between our results and those from Ghia et al. (1982) is quite good, although neither upwind technique nor second order time integration have been used. The mesh for discretization of the domain was of 161 by 161 grid points with a total of 64964 variables. The mesh in this work was a few more refined than that of Ghia and coworkers, however, for low Reynolds numbers, even a coarse mesh yields results in good agreement with results from the literature.

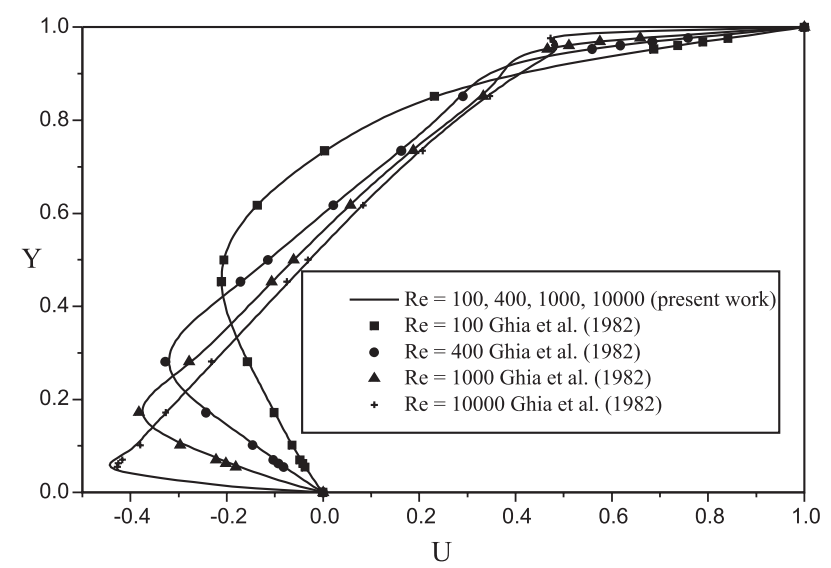

Figure 4. U-velocity at $\mathrm{X}=0.5$

The stream functions are shown in Figs. 6-8 for three Reynolds numbers, 400, 1,000 and 10,000, respectively. The secondary vortices at the corners of the cavity have been satisfactorily predicted. The numerical values of the stream functions, not shown in these figures, are in good agreement with the results from Ghia et al. (1982).

\section{CONCLUSIONS}

In this work we have presented a large-eddy simulation of incompressible fluid by a control volume-finite element method using nine-node finite elements with quadratic and linear interpolation functions for the velocity and pressure fields, respectively. The benchmark problem of the lid-driven square cavity has been solved in a personal computer Pentium 4, $2 \mathrm{GHz}$ and $2 \mathrm{~Gb}$ of RAM. The results were compared with available results from the literature and a good agreement was found. No upwind technique has been applied and a totally implicit method was used for time discretization. The main contribution of this work was an implementation of LES with the sub-domain finite element method. Turbulence is a 3D phenomenon, however, only 2D simulations have been done.

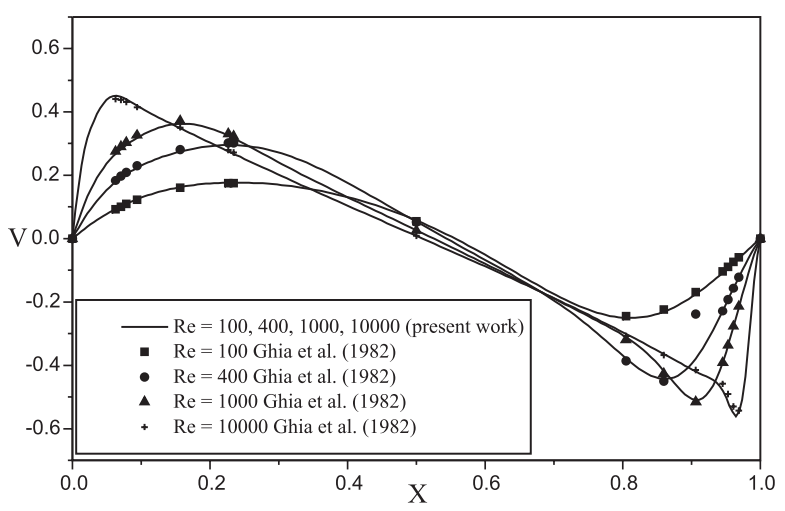

Figure 5. V-velocity at $\mathrm{Y}=0.5$

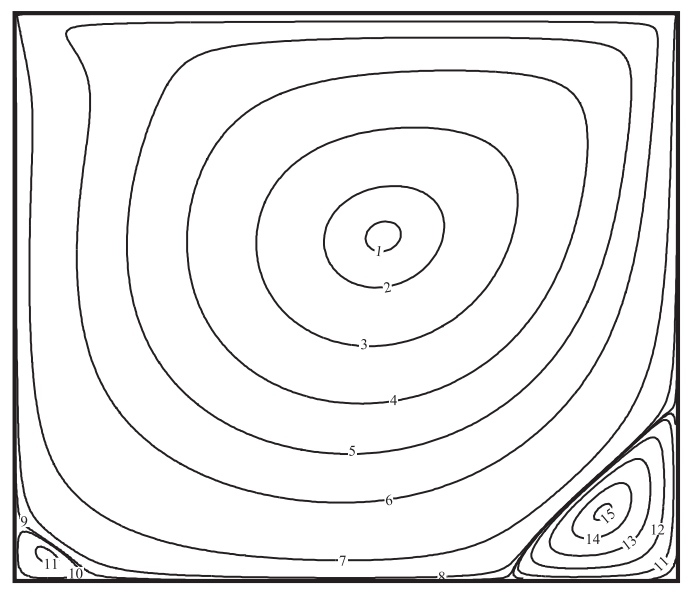

Figure 6. Stream function for $\mathrm{Re}=400$ 


\section{CIÊNCIA/SCIENCE}

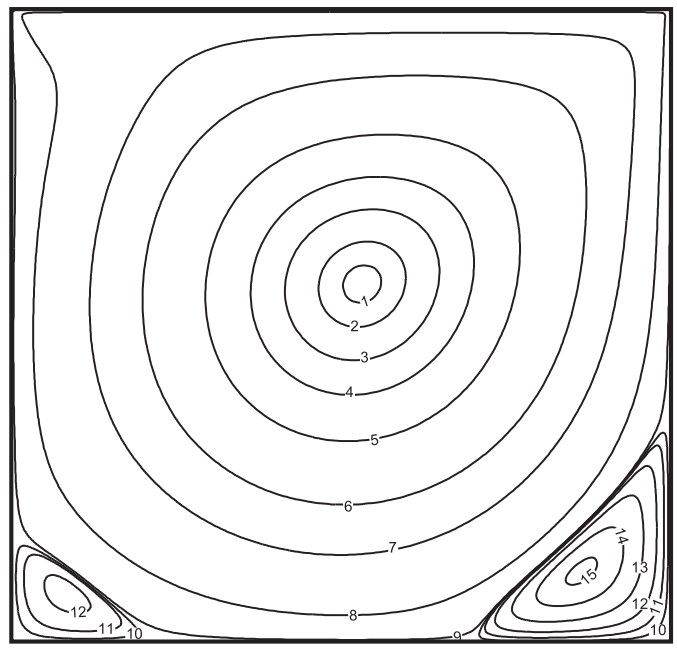

Figure 7. Stream function for $\mathrm{Re}=1,000$

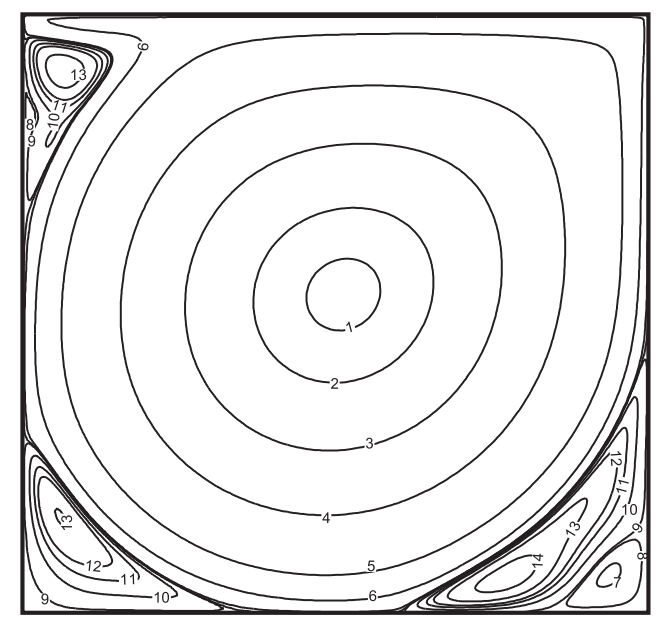

Figure 8. Stream function for $\operatorname{Re}=10,000$

The natural sequence of this work is the three-dimensional simulations and the use of dynamic models to compute the constant of Smagorinsy. Some results for regions with inflow and outflow have been already obtained and they encourage the continuity of this work.

\section{ACKNOWLEDGEMENTS}

The authors would like to acknowledge FAPESP for the computational resources and FUNDUNESP for the grants related to the development of this work.

\section{REFERENCES}

Almeida., O., 2001, Numerical Simulation of Large Scales of Transitional Flows around Cylinders of Square and Rectangular Base, Master Dissertation UNESP-Ilha Solteira, Ilha Solteira, SP, Brazil. (in Portuguese)
Baliga, B. R., and Patankar, S. V., 1980, A New Finite-element Formulation for Convection Diffusion Problems, Numerical Heat Transfer, Vol. 3, pp. 393-409.

Baliga, B.R., and Patankar, S. V., 1983, A Control Volume Finite-element Method for Two Dimensional Fluid Flow and Heat Transfer, Numerical Heat Transfer, Vol. 6, pp. 245-261.

Banaszek, J., 1989, Comparison of Control Volume and Galerkin Finite Element Methods for Diffusion-type Problems, Numerical Heat Transfer, Vol. 16, pp. 59-78.

Campregher Jr., R., 2002, Numerical Simulation of Transitional and Turbulent Flows around Cartesian Geometries, Master Dissertation, UNESP Ilha Solteira, Ilha Solteira, SP, Brazil. (in Portuguese)

Chidambaram, N., 1998 Colocated-grid Finite Volume Formulation for the Large Eddy Simulation of Incompressible and Compressible Turbulent Flows. M.Sc. Dissertation. Graduate College, Department of Mechanical Engineering, Iowa State University, Ames, Iowa, USA.

Dhatt, G., and Touzot, G., 1984, Une Presentation de la Méthode des Éléments Finis, Deuxieme Édition, Maloine S.A. Éditeur, Paris. (in French)

Galpering, B., and Orszag, S. A., 1993, Large Eddy Simulation of Complex Engineering and Geophysical Flows, Cambridge University Press.

Ghia, U., Ghia, K. N., and Shin, C. T., 1982, High-Re Solutions for Incompressible Flow Using the Navier-Stokes Equations and a Multigrid Method, Journal of Computational Physics, Vol. 48, No. 3, pp. 387-411.

Jiang, B-N., 1998. The Least-Squares Finite Element Method: Theory and Applications in Computational Fluid Dynamics and Electromagnetics. Springer-Verlag.

Matos, A., Pinho, F. A. A., and Silveira Neto, A., 1999, Large-Eddy Simulation of Turbulent Flow over a Two-Dimensional Cavity with Temperature Fluctuations. International Journal of Heat and Mass Transfer, Vol. 42, pp. 49-59.

Padilla, E. L .M., and Silveira_Neto, A., 2001, Large-Eddy Simulation of Transitional Natural Convection over a Horizontal Heated Cylinder, Using a Dynamic Sub-Grid Scale Model. In: $2^{\text {nd }}$ International Conference on Computational Heat and Mass Transfer. Rio de Janeiro/RJ. 
Raw, M. J., and Schneider, G. E., 1986, A Skewed, Positive Influence Coefficient Up-Winding Procedure for Control-Volume-Based FiniteElement Convection-Diffusion Computation. Numerical Heat Transfer, Vol. 9, pp. 1-26.

Raw, M. J., Schneider, G. E., and Hassani, V., 1985, A 9-Noded Quadratic Control Volume Based Finite Element for Heat Conduction, Journal of Spacecraft and Rockets, Vol. 22, No. 5, pp.523-529.

Saabas, H. J., and Baliga, B. R., 1994a, Colocated Equal-order Control-volume Finite-element Method for Multidimensional, Incompressible, Fluid Flow - Part I: Formulation, Numerical Heat Transfer, Part B, Vol. 26, pp. 381-407.

Saabas, H. J., and Baliga, B. R., 1994b, Colocated Equal-order Control-volume Finite-element Method for Multidimensional, Incompressible, Fluid Flow - Part II: Verification, Numerical Heat Transfer, Part B, Vol. 26, pp. 409-424.

Silva, J. B. C., 1998, Numerical Simulation of Fluid Flow by the Finite Element Method Based on Control Volumes, Doctoral Thesis, FEMUNICAMP, Campinas, São Paulo, Brazil. (in Portuguese)

Silva, J. B. C., and Moura, L. F. M., 1997, Numerical Simulation of Fluid Flow by the Control Volume-finite Element Method. In: XIV Brazilian Congress of Mechanical Engineering - COBEM (in CD-ROM), Bauru-SP, Brazil, 8p.

Silva, J. B. C., and Moura, L. F. M., 2001, A Control-Volume-Finite-Element Method (CVFEM) for Unsteady, Incompressible, Viscous Fluid Flows. Numerical Heat Transfer Part B: Fundamentals, Vol. 40, No. 1, pp 61-82.

Silva, J. B. C., Vieira, E. D. R., and Moura, L. F. M., 1998, Control Volume-finite Element and Flow Visualization Methods Applied for Unsteady Viscous Flow Past a Circular Cylinder. In: V Congresso de Engenharia Mecânica do NorteNordeste, Fortaleza, CE, Brazil, Vol. II, pp. 80-87.

Silva, J. B. C., Aparecido, J. B., and Moura, L. F. M., 1999, A Control Volume-Finite Element Method (CVFEM) for Unsteady Fluid Flows. In: XV Brazilian Congress of Mechanical Engineering - COBEM (in CD-ROM), Águas de Lindóia/SP.

Taylor, C., and Hughes, T. G., 1981, Finite Element Programming of the Navier-Stokes Equations, Pineridge Press Ltd, Swansea, U.K.

Wilcox, D. C., 1993, Turbulence Modeling for CFD. DCW Industries, Inc. La Cañada, California, USA. 